\title{
Recurrence Formula of the Taylor Series Expansion Coefficients of the Jacobian Elliptic Functions
}

\section{By Alois Schett}

\begin{abstract}
A general recurrence formula permitting calculation of the Taylor series expansion coefficients of the Jacobian elliptic functions and the number of permutations of $n$ natural numbers with a given run up or peak is given and its application is demonstrated.
\end{abstract}

In [1] we studied properties of the Taylor series expansion coefficients $A_{n}$ comprising those of the Jacobian elliptic functions and tabulated them up to $n=15$. Further tabulations of these coefficients for $n=16$ to $n=50$ are published in [2] . In the present paper we are giving a recurrence formula for the coefficients $A_{n}$.

We recapitulate briefly for later use the properties of $A_{n}$ studied in [1]:

1. $A_{n}$ are triangle matrices with $\left(n_{\text {even }}+2\right) / 2$ or $\left(n_{\text {odd }}+1\right) / 2$ columns and rows.

2. $A_{n}=A_{n}^{T}$.

3. The sum of the elements of $A_{n}$ is equal to $n$ !.

4. The sum of the elements of a row $i$ of $A_{n}$ is the number of permutations of $n$ natural numbers with $i-1$ runs up.

5. The sum of the elements $a_{i, j}$ of $A_{n}$ with $i+j$ constant $>m$ ( $m$ maximal rows) is the number of permutations of $n$ natural numbers with $k=n-(i+j)-1$ peaks.

6. For $n$ even and $i+j=(n+2) / 2+1,\left(a_{i, j}\right)_{n}=\left(a_{i, j}\right)_{n+1}$. For $n$ odd $a_{i,(n+1) / 2}=a_{i,(n+3) / 2}$.

7. $a_{(n+2) / 2,(n+2) / 2}=0$ for $n$ even. $a_{(n+1) / 2,(n+1) / 2}=2^{n-1}$ for $n$ odd.

8. The elements $\left(a_{i, j}\right)_{n}, i+j=(n+2) / 2+1$, and $\left(a_{i,(n+2) / 2}\right)_{n}$ ( $n$ even) are the Taylor series expansion coefficients of the Jacobian elliptic functions $\operatorname{sn}(u, k)$ and $\operatorname{cn}(u, k), \operatorname{dn}(u, k)$, respectively.

The formal recurrence formula for $A_{n}$ reads $A_{n+1}=T_{n} A_{n}$. We have to find $T_{n}$ and to define its application on $A_{n}$. By means of mathematical induction we obtained the following results.

$T_{n}$ are triangle matrices with the elements

$$
\left(t_{i, j ; 1}, t_{i, j ; 2}, t_{i, j ; 3}\right)_{n}=(n-2(j-1), 3+2(j-1)-n+2 i, n+2-2 i)_{n},
$$




$$
\begin{array}{r}
\left(t_{i, j ; 1}, t_{i, j ; 2}, t_{i, j ; 3}\right)_{n}=(0,0,0)_{n} \text { for } i+j<n / 2+1, n \text { even and } \\
i+j<(n-1) / 2+1, n \text { odd }
\end{array}
$$

$i ; j=1,2,3, \ldots, n / 2$ for $n$ even and $(n-1) / 2$ for $n$ odd.

The symmetry

$$
\left(t_{i, j ; 1}, t_{i, j ; 2}, t_{i, j ; 3}\right)=\left(t_{j, i ; 3}, t_{j, i ; 2}, t_{j, i ; 1}\right)
$$

and the relation

$$
t_{i, j ; 1}+t_{i, j ; 2}+t_{i, j ; 3}=n+5
$$

are valid.

Applying $\left(t_{i, j ; 1}, t_{i, j ; 2}, t_{i, j ; 3}\right)_{n}$ on $\left(a_{n, k}\right)_{n}$ according to the relation

$$
\left(a_{i, j}\right)_{n+1}=\left(a_{i, j-1} \cdot t_{i-1, j-1 ; 1}+a_{i, j} \cdot t_{i-1, j-1 ; 2}+a_{i-1, j} \cdot t_{i-1, j-1 ; 3}\right)_{n}
$$

and using the properties 2. and 6. of $A_{n}$ mentioned above permits calculation of the elements of $A_{n+1}$.

Examples. We illustrate these formulas on $n$ even and $n$ odd.

$$
\begin{aligned}
& A_{7}=T_{6} A_{6}, \\
& A_{6}=\left(\begin{array}{cccc}
0 & 0 & 0 & 1 \\
0 & 0 & 135 & 44 \\
0 & 135 & 328 & 16 \\
1 & 44 & 16 & 0
\end{array}\right), \quad T_{6}=\left(\begin{array}{lll}
0,0,0,0,0,02,3,6 \\
0,0,0,4,3,4 & 2,5,4 \\
6,3,2 & 4,5,22,7,2
\end{array}\right), \\
&\left(a_{2,4}\right)_{7}=\left(a_{2,3} \cdot t_{1,3 ; 1}+a_{2,4} \cdot t_{1,3 ; 2}+a_{1,4} \cdot t_{1,3 ; 3}\right)_{6} \\
&=135 \cdot 2+44 \cdot 3+1 \cdot 6=408, \\
&\left(a_{3,4}\right)_{7}=\left(a_{3,3} \cdot t_{2,3 ; 1}+a_{3,4} \cdot t_{2,3 ; 2}+a_{2,4} \cdot t_{2,3 ; 3}\right)_{6} \\
&= 328 \cdot 2+16 \cdot 5+44 \cdot 4=912, \\
&\left(a_{4,4}\right)_{7}=\left(a_{4,3} \cdot t_{3,3 ; 1}+a_{4,4} \cdot t_{3,3 ; 2}+a_{3,4} \cdot t_{3,3 ; 3}\right)_{6} \\
&=16 \cdot 2+0 \cdot 7+16 \cdot 2=64 \\
&\left(a_{3,3}\right)_{7}=\left(a_{3,2} \cdot t_{2,2 ; 1}+a_{3,3} \cdot t_{2,2 ; 2}+a_{2,3} \cdot t_{2,2 ; 3}\right)_{6} \\
&=135 \cdot 4+328 \cdot 3+135 \cdot 4=2064 .
\end{aligned}
$$

According to property 6 we obtain $a_{1,4}=1, a_{2,3}=135$, and since $A_{n}$ is symmetric all elements of $A_{7}$ are known.

$$
A_{8}=T_{7} A_{7}
$$




$$
\begin{aligned}
& A_{7}=\left(\begin{array}{cccc}
0 & 0 & 0 & 1 \\
0 & 0 & 135 & 408 \\
0 & 135 & 2064 & 912 \\
1 & 408 & 912 & 64
\end{array}\right), \quad T_{7}=\left(\begin{array}{ccc}
0,0,0 & 0,0,0 & 3,2,7 \\
0,0,0 & 5,2,5 & 3,4,5 \\
7,2,3 & 5,4,3 & 3,6,3
\end{array}\right) \\
&\left(a_{2,4}\right)_{8}=\left(a_{2,3} \cdot t_{1,3 ; 1}+a_{2,4} \cdot t_{1,3 ; 2}+a_{1,4} \cdot t_{1,3 ; 3}\right)_{7} \\
&=135 \cdot 3+408 \cdot 2+1 \cdot 7=1228 \\
&\left(a_{3,4}\right)_{8}=\left(a_{3,3} \cdot t_{2,3 ; 1}+a_{3,4} \cdot t_{2,3 ; 2}+a_{2,4} \cdot t_{2,3 ; 3}\right)_{7} \\
&=2064 \cdot 3+912 \cdot 4+408 \cdot 5=11880, \\
&\left(a_{4,4}\right)_{8}=\left(a_{4,3} \cdot t_{3,3 ; 1}+a_{4,4} \cdot t_{3,3 ; 2}+a_{3,4} \cdot t_{3,3 ; 3}\right)_{7} \\
&=912 \cdot 3+64 \cdot 6+912 \cdot 3=5856, \\
&\left(a_{3,3}\right)_{8}=\left(a_{3,2} \cdot t_{2,2 ; 1}+a_{3,3} \cdot t_{2,2 ; 2}+a_{2,3} \cdot t_{2,2 ; 3}\right)_{7} \\
&=135 \cdot 5+2064 \cdot 2+135 \cdot 5=5478
\end{aligned}
$$

Using the properties 6 . and 2. of $A_{n}$, we obtain the remaining elements of $A_{8}$.

$$
A_{8}=\left(\begin{array}{ccccc}
0 & 0 & 0 & 0 & 1 \\
0 & 0 & 0 & 1228 & 408 \\
0 & 0 & 5478 & 11880 & 912 \\
0 & 1228 & 11880 & 5856 & 64 \\
1 & 408 & 912 & 64 & 0
\end{array}\right)
$$

In conclusion, the present recurrence formula permits calculation of the Taylor series expansion coefficients of the Jacobian elliptic functions and the number of permutations of $n$ natural numbers with a given run up or peak.

Centre d'Etudes Nucléaires

Saclay, France

1. A. SCHETT, "Properties of the Taylor series expansion coefficients of the Jacobian elliptic functions,” Math. Comp., v. 30, 1976, pp. 143-147. MR 52 \#12298.

2. A. SCHETT, Addendum to "Properties of the Taylor series expansion coefficients of the Jacobian elliptic functions," Microfiche supplement, Math. Comp., v. 31, 1977, no. 137. 